for several dates, he publishes a series of photographs of its form, displaying the remarkable changes which the tail underwent.

Franklin Adams Chart of the Sky.-The Royal Astronomical Society has undertaken the publication of a limited number of reproductions of the FranklinAdams chart. The 206 sheets form a complete map of the whole sky, the area of each being $15^{\circ}$ by $15^{\circ}$. It will be remembered that the original plates were secured with a ro-in. Cooke triplet objective of 45 in. focal length; the negatives show stars down to the sixteenth and seventeenth magnitudes. The reproductions will be on bromide paper, $I_{5}$ by $I_{2}$ in., the chart area being II by $x$ I in. The complete price will be ten guineas, and it is expected that the first sets will be ready for delivery in twelve months' time. It is hoped that a sufficient number of subscribers will be enlisted to help to defray the cost of such an expensive undertaking.

A Cheap Form of Grating Spectrograph.-In the current number of Knowledge (vol. xxxvi.; No. 537, p. 142) Mr. A. H. Stuart describes what seems to be a new form of spectroscope in which a transmission grating is used. The instrument is there illustrated by two diagrams, and the principle involved can be easily grasped. The instrument is of the rectangular box form, having the slit and camera at one end of the box. The light, after passing through the slit, falls on an objective, at the back of and nearly in contact with it being placed a replica grating; behind this grating is placed a plane mirror at a distance of a few inches. The beam of light passes through the slit to the objective, and falls normally on the grating. A large portion of the light passes through the grating unchanged, and falls on the mirror. If it meets the mirror normally it will be reflected back to the grating, and a spectrum will pass out obliquely through the object glass and fall on the photographic plate at the camera end to one side of the collimator. In order to avoid the faint reflection spectrum the grating is retained in its position at right angles to the incident beam, but the mirror is slightly twisted. Thus a pure spectrum of considerable dispersion is obtained. Mr. Stuart has constructed such an apparatus by the judicious use of $20 s$., the achromatic lens, 2 in. in diameter, costing $3^{s} .6 d$, and the grating Ios. $6 d$.

Khedivial Observatory, Helwan.-Two bulletins, Nos. 8 and 9 , from this observatory indicate the useful astronomical work that is being accomplished in Egypt. The first gives an account of the method adopted and the results obtained in determining the astronomical positions of E1 Daba'a, Mersa Malrûh, Baqbaq, Sollûm, and Siwa. The work was carried out by Messrs. E. B. H. Wade and H. Knox Shaw. The second of the two bulletins contains the results of the first three years (I909-II) of nebular photography with the Reynolds reflector obtained by $\mathrm{Mr}$. H. Knox Shaw. It is stated that during this period the instrument was constantly undergoing alterations and repairs, so that some of the plates are not so good as they might be. Nevertheless, some of them afford considerable information as to the structure of some nebulæ not hitherto photographed. The table gives the new general catalogue numbers, the positions for I 900 and remarks, and four plates, each containing four or more reproductions, conclude the publication. Attention is directed to the advantage of making drawings of the smaller and less brilliant nebulæ from the negatives, a method which is capable of reproducing the general form of the nebula almost as accurately as any photographic reproduction.

\section{THE DEVELOPMENT OF THE PARASITE OF INDIAN KALA-AZAR.}

$\mathrm{I}$ a recent memoir with the above title, ${ }^{1}$ Captain W. S. Patton gives a detalled account of invescigations carried on by him in Madras upon the development and transmission of the parasite of Kala-azar, commonly known as Leishmania donovani. As the result of numerous experiments with various blood-sucking insects, the author concludes that the transmission of Indian Kala-azar from man to man is effected solely by bed-bugs of the genus Cimex, and finds that the parasite develops as readily in $C$. lectularius, the species common in Europe, as in the Indian species, c. rotundatus. The development observed by the author takes place entirely in the digestive tract of the bug, and is in the main as follows.

The bug takes up the parasite from an infected person in the leishmanial form, that is to say, as the familiar "Leishman-Donovan body," contained either within white blood-corpuscles or in macrophages, in the peripheral blood. After being ingested by the bug, the parasites remain in an unchanged condition for some thirty-six to forty-eight hours. The earliest developmental changes in the gut of the bug may take place while the parasite is still enclosed in a leucocyte or after it has been set free by disintegration of the host-cell, and consist of an increase in the size of the parasite, with enlargement of its trophic and kinetic nuclei. As growth proceeds, the parasites may multiply by binary fission.

The next event in the development of the parasite is the formation of a flagellum, which takes place from the third to the fifth day after the last feed of infected blood. A young, growing parasite may, without dividing, become elongated and spindle-shaped, and acquire a flagellum; or it may first multiply by binary fission, after which each of the two daughter individuals acquires a flagellum; or the parasite may go through a process of multiple fission, in which the two nuclei, trophic and kinetic, divide each into eight or more, and as many flagella grow out, with subsequent division of the body into a number of flagellated daughter-individuals. However the details of the process may vary, the final result is the same, and by the fifth day the parasites, considerably increased in number, have the form of long, actively moving flagellates of the Herpetomonas type, familiar to all those who have studied the development of the parasite in artificial cultures ever since these changes were first discovered and described by Rogers.

About the sixth or seventh day the flagellate para sites are observed to be attaching themselves by their flagella to the intestinal wall of the bug. When thus attached, the body of the parasite slowly rounds up and at the same time it divides; the smaller forms thus produced divide again, and meanwhile the flagellum becomes shorter, and finally disappears altogether. The result of these changes is that the parasite reverts again from the herpetomonad phase to the form of the small, non-flagellate leishmanial body, distinguished by the author as the "post-flagellate" phase, though it does not appear to differ in any essential detail from the initial "preflagellate" leishmanial form, but is described as having a distinct envelope ("periplast"). The post-flagellate stage in the bug begins about the eighth day, and is completed by the twelfth.

According to Captain Patton, this post-flagellate stage represents the final stage of the development of the parasite in the bug. $\mathrm{He}$ 1 Scientific Memoirs by Officers of the Medical and Sanitary Depart-
ments of the Government of India. No. 53, "The Development of the Parasite of Indian Kala-Azar." P. $\mathrm{p}+38+\mathrm{r}$ plate. (Calcutta: Government Printing Office, Igrz.) Price Is. $2 d$. 
believes that the post-flagellate phase finds its way back again to the human being, when the bug feeds again, by regurgitation from the intestine. Proof of this is as yet lacking, but he hopes in future experiments to solve this part of the problem once and for all. He states that if the bug takes a fresh feed of blood when the parasites in its intestine are in the flagellated phase, they are all destroyed, and cannot develop further. "Human blood has some body in it which not only prevents the process of flagellation, but also destroys the flagellates. This substance is in all probability the complement, and it is known that it is itself destroyed in about two days, when blood is drawn from the human body. This fact further explains why the parasite only begins its development in the bug on the second day. ... Though many bugs may become infected, only those which do not feed again till the parasite has passed back to its post-flagellate stage are infective." $\mathrm{He}$ believes that only in its "post-flagellate" leishmanial form can the parasite resist the destructive action of fresh blood and re-infect the vertebrate host.

The author's conclusion that a non-flagellate leishmanial stage is the final phase in the development of the parasite in the insect-host is based largely on a comparison with the herpetomonad parasites of insects; that is to say, on an analogy with species which are parasitic in invertebrate hosts alone and have no alternate vertebrate host in their life-cycle. In such species, however, the infection of new hosts is effected (apart from the possible occurrence of "hereditary" infection) by the contaminative method; that is to say, by means of resting, non-flagellate phases, usually encysted, which pass out of the host in the fæces, and are accidentally swallowed by another insect-host. On the other hand, in all known cases where a flagellate parasite has an alternation of hosts, vertebrate and invertebrate, and where the vertebrate host is infected by the inoculative method, that is to say, by the parasite being injected into it through the mouthparts of the invertebrate in the act of sucking blood; in all such cases that have been investigated accurately up to the present, the final stage of the parasite in the invertebrate host is an active flagellate. Further, it has been frequently observed in, for instance, the development of trypanosomes in their alternate invertebrate hosts that the active, flagellate forms, usually crithidial or herpetomonad in type, may pass temporarily into a resting, non-flagellate, leishmanial phase during hunger-periods, when the ingested blood is digested and absorbed, and become active flagellate forms again when the host takes in a fresh supply of food.

From these considerations the possibility is not to be excluded at present that Captain Patton's observations may be capable of an interpretation different from that which he places upon them. It may well be that his "post-flagellate" stage represents a resting phase upon which the parasite enters when the blood taken up by the bug is digested, and that when the bug feeds again these resting forms will become active once more, and give rise to a final flagellate stage, yet to be discovered, which will be inoculated ultimately into the human being. It must, however, be borne in mind that it has not yet been proved definitely that the parasite passes from the bug into the human being by inoculation through the proboscis; if, as is at least possible, the parasite is destined to pass out of the bug in its fæces, it is then probable in the highest degree that the final stage in the development in the bug would be a resting, non-flagellate phase.

The final decision, however, with regard to the transmission of the parasite of Kala-azar will rest, not upon analogies with other parasites, but upon facts demonstrated with regard to this parasite itself, and if Captain Patton establishes his statements, he will have added a new type of development and transmission to those known already to occur in flagellate parasites of vertebrates transmitted by blood-sucking invertebrates. However this may be, the author is to be congratulated on having brought forward very strong evidence to show that, as suggested originally by Rogers, the spread of this very deadly human disease is to be attributed to the agency of the bedbug, a discovery of immense practical importance.

E. A. Minchin.

\section{NEW ZEALAND VEGETATION.'}

I $\mathrm{N}$ a brief general account, contributed to the "New Zealand Year-book, rgiz," Dr. L. Cockayne, F.R.S., who has done so much floristic and ecological work in New Zealand, points out that owing to its long isolation and diverse elements (Malayan, Australian, subantarctic, and endemic), the flora of New Zealand is of special interest.

The vascular plants in this flora-ferns, fern-allies, and seed-plants--number, so far as at present known, about I7oo species, of which about three-fourths are endemic. Of the lower plants-algæ, fungi, lichens, liverworts, and mosses-many hundreds have been described, including many remarkable genera and species, but there can be no doubt that hundreds more remain to be described. The ferns and fernallies form a striking feature in the vegetation in some areas, but are not of such great relative importance in the New Zealand flora as has sometimes been supposed; still, about I6o species of these plants are known.

Among the seed-plants, the daisy family is the largest, as might be expected, having more than 230 species; the sedge, grass, and figwort families follow with more than Ioo species each, while between thirty and seventy species belong in each case to the orchid, carrot, buttercup, bedstraw, epacrid, willowherb, pea, rush, and forget-me-not families.

Among genera which contain many species and are marked by great variability, making them difficult to define and classify, Dr. Cockayne mentions Veronica, Carex, Ranunculus, Senecio, Epilobium, and Myosotis. The genus Veronica, with more than IOO species, is remarkable for its variability and for the almost endless variety of habit assumed by the various species, some of the New Zealand speedwells (mostly endemic and largely alpine in habitat) being small trees, while the majority are shrubby and often dwarf, frequently simulating cypresses and other conifers owing to their reduced and appressed leaves. Apart from variability in the adult plants, about Ioo New Zealand species, belonging to different genera, have juvenile forms which are quite distinct from the adult forms, and may retain their juvenile characters for many years; this is seen in various trees, such as lace-bark, lancewood, and ribbonwood.

Among the multitudinous growth-forms, characteristic of diverse life conditions, the more remarkable are the climbers with woody rope-like stems, resembling the lianas of the South American tropical forests; shrubs with wiry interlaced branches forming close masses; the curious cushion-plants, sometimes of immense size, as in the vegetable sheep (species of Haastia, Raoulia, and Psychrophyton); leafless shrubs with round or flattened stems, and so on. The woody plants are almost all evergreen, only some twenty species being deciduous or semi-deciduous; herbs that

1 The Flora of New Zealand." By Dr. L. Cockayne. Extract from the "New Zealand Year-book, Igr2." 